\title{
Spherical and Cylindrical Ion Acoustic Solitary Waves in Electron-Positron-Ion Plasmas with Non-Maxwellian Electrons and Positrons
}

\author{
Sukhjeet Kaur ${ }^{1}$, Parveen Bala ${ }^{2}$ \\ ${ }^{1,2}$ (Department of Mathematics, Statistics and Physics, Punjab Agricultural University, Ludhiana, India)
}

\begin{abstract}
The propagation of cylindrical and spherical ion acoustic solitary waves in a plasma system consisting of ions, electrons and positrons are investigated. The electrons and positrons are assumed to be following the nonextensive distribution popularly known as Tsallis distribution. The standard nonlinear equation i.e. Korteweg de-Vries (KdV) equation has been solved numerically using suitable mathematical transformations. The effect of nonextensivity $(q)$ and nonplanar geometry on the amplitudes and width of ion acoustic potential structures have been studied numerically. A transition from negative to positive potential structures have been observed for the planar as well as nonplanar geometries for lower values of $q$ in the range $-1<q<0$. Soliton amplitude is maximum for spherical waves and is minimum and for planar waves while it lies in between the two for cylindrical waves. The present investigation may help us in understanding the study of cylindrical and spherical solitary waves in astrophysical plasmas.
\end{abstract}

Keywords: Cylindrical structures, Electron-positron-ion (e-p-i) plasmas, Reductive perturbation method, Spherical structures, Tsallis distribution.

\section{Introduction}

The theoretical and experimental study of the dynamics of ion acoustic waves (IASWs) is of great interest for several decades. Many researchers and scholars have done much work on IASWs in different plasma systems. A solitary wave is a nonlinear wave having permanent shape and it maintains its shape during its propagation. The word acoustic is referred to as sound so these ion acoustic waves are also called as sound waves. It arises because of the balance between the effects of the nonlinearity and the dispersion. Ikezi et al [1] have done the first experimental observation of ion-acoustic solitons. After that many researchers [2-8] have reported these solitons with their theoretical work in different plasma systems. Much study of ion acoustic solitons (IASs) has been reported in electron-positron-ion (e-p-i) plasmas by a lot of researches during past few years [9-17]. Electron-positron-ion plasmas are composed of a small number of ions along with the electrons and positrons. An e-p-i plasma with positive ions is believed to be widespread in nature. Such a plasma can occur, e.g., in the inner region of accretion discs, in the vicinity of black holes [18-19], in magnetospheres of neutron stars [20-21], in active galactic cores [22], and even in solar flare plasma [23]. Recently, narrowcollimated extended relativistic jets of e-p-i plasma were observed in the vicinity of blazars and micro quasars [24-26]. There are many facts indicating that our Universe was a hot e-p-i plasma during the first minutes of its existence [27]. For laboratory plasmas, it is known that the propagation of a short relativistically strong laser pulse in matter can be accompanied by the formation of e-p-i plasma due to photoproduction of pairs during photons scattering by nuclei, etc. [28-30]. Tokamaks and other magnetic confinement systems are also another examples related to plasma. To determine the rate of transport of particle from the positron lifetime the annihilation line in the $\gamma$ spectrum, charged positron or quasineutral electron-positron beams can be injected into such plasmas [31-32]. In this case, a small region occupied by e-p-i plasma can form in the bulk plasma. These mainly exist in astrophysical environments. So the study of linear and nonlinear dynamics in e-p-i plasmas are quite important and of great interest. With addition to these plasma systems a great attention has been paid to nonextensive statistic mechanics. It is derived from the main statistics i.e. Boltzmann-Gibbs-Shannon (BGS) statistics. As the Maxwell distribution is proved unsuitable for study of many plasma systems so Tsallis [33] introduced another generalization called the non-Maxwellian distribution in 1988. The main credit for this new generalization firstly goes to Renyi [34]. Nonlinearity plays an important role in plasma because it is based on nonlinear dynamics and having nonlinear equations. Therefore many researchers have been studied the spherical and cylindrical (nonplanar) modes of ion acoustic waves and compared the results of these geometries with the planar ones [35-38]. Stephen Maxon [38] studied the cylindrical and spherical waves and proposed that these are two different waveforms having different properties. The properties of these higher dimensional solitons are quite different from their planar counterpart. Javidan [39] has derived the cylindrical and spherical KdV equation for ion acoustic solitary waves in e-p-i plasmas using kappa distribution. Only few investigations have been reported on the study of nonplanar ion acoustic solitary waves in e-p-i plasmas [40-42]. Sahu and 
Roychoudhury [42] investigated the exact solutions of cylindrical and spherical dust-ion acoustic waves. For this they derived the nonplanar $\mathrm{KdV}$ equation by using a suitable coordinate transformation which reduces the cylindrical $\mathrm{KdV}$ equation into the ordinary $\mathrm{KdV}$ equation which can be solved analytically. Eslami and Mottaghizadeh [43] used the standard reductive perturbation technique, a three-dimensional cylindrical Kadomtsev-Petviashvili equation (CKPE), which governs the dynamics of ion acoustic solitary waves (IASWs), is derived for small but finite amplitude ion-acoustic waves in cylindrical geometry in a collisionless unmagnetized plasma with kappa distributed electrons, thermal positrons, and cold ions. Electron-positron (e-p) pairs exist in the plasmas emanating both from the pulsars and from the inner region of the accretion discs surrounding the central black hole in active galactic nuclei [44-46]. Such pairs are also present in the Van Allen radiation belt and near the polar cap of fast rotation neutron stars [47-50], in semiconductor plasma [51], in intense laser fields [28], in tokamaks [52] and in the solar atmosphere [53]. It has been found that the electron positron (e-p) plasmas behave differently from typical electron-ion (e-i) plasmas [54]. In astrophysical environments always there exist a small number of ions with electrons and positrons, it is then important to study the linear and nonlinear dynamics of plasma waves in electron-positron-ion (e-p-i) plasmas. A number of works has been carried out to study the linear and nonlinear behaviors of waves in e-p and e-p-i plasmas over the last few years [55-59]. Based on Maxwellian assumption, many authors have been studied the propagation of ion acoustic waves in electron-positron-ion plasmas [60-63].

In this paper, we investigate the propagation of cylindrical and spherical ion acoustic solitary waves in a plasma system consisting of ions, electrons and positrons. The electrons and positrons are assuming to be following the nonextensive distribution popularly known as Tsallis distribution. The standard nonlinear equation i.e. Korteweg de-Vries (KdV) equation has been solved numerically using suitable mathematical transformations. The effect of nonextensivity $(q)$ and nonplanar geometry on the amplitudes and width of ion acoustic potential structures have been studied numerically.

\section{Governing Equations And Derivation of KdV Equation}

We focus on the cylindrical and spherical ion acoustic solitary waves in plasmas containing cold ion fluid, nonextensively distributed electrons and positrons. In equilibrium, the charge neutrality condition is $n_{e 0}=n_{i 0}+n_{p 0}$. Where $n_{i 0}, n_{p 0}$ and $n_{e 0}$ are the unperturbed number densities of the ion, positron and electron respectively. The nonlinear dynamics of IAWs in cylindrical and spherical geometries is governed by the following set of nonlinear equations

$$
\begin{aligned}
& \frac{\partial n}{\partial t}+\frac{1}{r^{m}} \frac{\partial\left(r^{m} n u\right)}{\partial r}=0 \\
& \frac{\partial u}{\partial t}+u \frac{\partial u}{\partial r}=-\frac{\partial \phi}{\partial r} \\
& \frac{1}{r^{m}} \frac{\partial}{\partial r}\left(r^{m} \frac{\partial \phi}{\partial r}\right)=n_{e}-n_{p}-n_{i}
\end{aligned}
$$

where $\mathrm{m}=0$ stands for one-dimensional flat geometry and $\mathrm{m}=1,2$ for cylindrical and spherical geometries respectively. The ion density $\left(n_{i}\right)$ and the ion velocity $\left(u_{i}\right)$ are normalized by the ion equilibrium density $n_{i 0}$ and the ion-acoustic speed $C_{s}=\sqrt{\frac{T_{e}}{m_{i}}}$ respectively, where $m_{i}$ is the ion mass. $\phi$ is electrostatic potential which is normalized by $\frac{T_{e}}{e}$ where $T_{e}$ is the electron temperature and "e" is the absolute value of electron charge. In the equations (1), (2) and (3), the densities of the plasma species are normalized by the unperturbed electron density $n_{e 0}$, space variable is normalized by the electron Debye length $\lambda_{D}=\sqrt{\frac{T_{e}}{4 \pi n_{e 0} e^{2}}}$ and time variable is normalized by the electron plasma period $T=\sqrt{\frac{m_{e}}{4 \pi n_{e 0} e^{2}}}$. The number density of electron and positron fluid, with nonextensive distribution is given by,

$$
\begin{aligned}
& n_{e}=\frac{1}{1-p}[1+(q-1) \phi]^{\frac{q+1}{2(q-1)}} \\
& n_{p}=\frac{p}{1-p}[1-(q-1) \sigma \phi]^{\frac{q+1}{2(q-1)}}
\end{aligned}
$$


Here $p=\frac{n_{p 0}}{n_{e 0}}$ is the ratio of unperturbed positron density to unperturbed electron density and $\sigma$ is the temperature ration of electrons to that of positron given by $\sigma=\frac{T_{e}}{T_{p}}$. Further the nonextensivity of electrons and positrons are assumed to be same. We use reductive perturbation technique (RPT) to derive the standard KdV equation. we introduce the stretched coordinates $\xi$ and $\tau$ as follows [64]

$\tau=\epsilon^{\frac{3}{2} t}, \quad \xi=-\epsilon^{\frac{1}{2}}(r+\lambda t)$

Here $\varepsilon$ is a small parameter and $\lambda$ is the wave phase velocity. The dependent variables expended as

$n_{i}=1+\varepsilon n_{i}^{(1)}+\varepsilon^{2} n_{i}^{(2)}+\varepsilon^{3} n_{i}^{(3)}+\cdots$

$u_{i}=\varepsilon u_{i}^{(1)}+\varepsilon^{2} u_{i}^{(2)}+\varepsilon^{3} u_{i}^{(3)}+\cdots$

$\phi=\varepsilon \phi^{(1)}+\varepsilon^{2} \phi^{(2)}+\varepsilon^{3} \phi^{(3)}+\cdots$

Substituting the stretching coordinates (6) and the expansions (7) into the basic equations (1), (2), (3) and (4-5) and comparing the coefficients of $\varepsilon$ after solving the equation, we have

$u_{i}^{(1)}=-\frac{\phi^{(1)}}{\lambda} \quad$ and $\quad n_{i}^{(1)}=\frac{\phi^{(1)}}{\lambda^{2}}$

To the next higher order, we get the phase velocity as

$\lambda=\sqrt{\frac{2(1-p)}{(1+p \sigma)(q+1)}}$

Finally after a tedious and long algebraic manipulation, we obtain a modified $\mathrm{KdV}$ equation

$\frac{\partial \phi^{(1)}}{\partial \tau}+\frac{m \phi^{(1)}}{2 \tau}+A \phi^{(1)} \frac{\partial \phi^{(1)}}{\partial \xi}+B \frac{\partial^{3} \phi^{(1)}}{\partial \xi^{3}}=0$

Where $\mathrm{A}$ is non-linear constant and $\mathrm{B}$ is dispersion constant given by

$A=\frac{1}{2}\left[\frac{3}{\lambda}-\frac{(q+1)(3-q)\left(1+p \sigma^{2}\right)}{8(1-p)}\right] \quad$ and $\quad B=\frac{\lambda^{3}}{2}$

Equation (10) is the cylindrical/spherical $\mathrm{KdV}$ equation describing the nonlinear propagation of the ion acoustic solitary waves in plasmas with nonextensive electrons. The coefficients A and B measures the nonlinearity and dispersive effects and their balance leads to the formation of localized wave known as soliton. Now we have to solve the final equation for its solutions for different values of $\mathrm{m}$.

For one dimensional flat geometry, $\mathrm{m}=0$. Equation (10) reduces to

$\frac{\partial \phi^{(1)}}{\partial \tau}+A \phi^{(1)} \frac{\partial \phi^{(1)}}{\partial \xi}+B \frac{\partial^{3} \phi^{(1)}}{\partial \xi^{3}}=0$

Here we use the transformation $\chi=\xi-u \tau$ and equation reduces to

$-u \frac{\partial \phi^{(1)}}{\partial \chi}+A \phi^{(1)} \frac{\partial \phi^{(1)}}{\partial \chi}+B \frac{\partial^{3} \phi^{(1)}}{\partial \chi^{3}}=0$

After solving, we have the solution of this equation is

$\phi^{(1)}=\phi^{(0)} \operatorname{Sech}^{2}\left(\frac{\chi}{\omega}\right)$

Where $\phi^{(0)}=\frac{3 u}{A}$ and $\omega=\sqrt{\frac{4 B}{u}}$

For nonplanar cylindrical geometry, $\mathrm{m}=1$

$\frac{\partial \phi^{(1)}}{\partial \tau}+\frac{\phi^{(1)}}{2 \tau}+A \phi^{(1)} \frac{\partial \phi^{(1)}}{\partial \xi}+B \frac{\partial^{3} \phi^{(1)}}{\partial \xi^{3}}=0$

To obtain an analytic solution of cylindrical KdV equation, let us first use Hirota's transformation [65] given by

$\phi=\frac{v}{\tau}+\frac{\xi}{2 A \tau}$

Under this transformation equation (14) transforms to

$\frac{\partial v}{\partial \tau}+\frac{A v}{\tau} \frac{\partial v}{\partial \xi}+\frac{\xi}{2 \tau} \frac{\partial v}{\partial \xi}+B \frac{\partial^{3} v}{\partial \xi^{3}}=0$

Again we use a transformation given by

$\tau^{\prime}=-2 \tau^{-1 / 2}, \quad \xi^{\prime}=\xi \tau^{-1 / 2}$

Equation (16) reduces to

$\frac{\partial v}{\partial \tau^{\prime}}+A v \frac{\partial v}{\partial \xi^{\prime}}+B \frac{\partial^{3} v}{\partial \xi^{\prime 3}}=0$

Above equation is the usual $\mathrm{KdV}$ equation. So the solution of this equation is given by $v=\phi^{(0)} \operatorname{Sech}^{2}\left(\frac{\chi}{\omega}\right)$ and the exact solution of equation (14) is given by 
$\phi_{2}=\frac{1}{\tau}\left\{\frac{\xi}{2 A}+\left(\frac{3 u}{A}\right) \operatorname{Sech}^{2}\left[\sqrt{\frac{u}{4 B \tau}}(\xi+2 u)\right]\right\}$

This solution is valid for $\tau \neq 0$.

For nonplanar spherical geometry, $\mathrm{m}=2$, the equation (10) reduces to $\frac{\partial \phi^{(1)}}{\partial \tau}+\frac{\phi^{(1)}}{\tau}+A \phi^{(1)} \frac{\partial \phi^{(1)}}{\partial \xi}+B \frac{\partial^{3} \phi^{(1)}}{\partial \xi^{3}}=0$

solution is given by

$\phi_{3}=\frac{1}{\tau}\left[\frac{\xi}{\operatorname{Aln} \tau}+\frac{c}{\ln \tau}\right]$

\section{Results And Discussion}

When the geometrical effect is taken into account (i.e. for $m \neq 0$ ), an exact analytical solution of equation (10) is not possible without any transformation. Therefore, we have solved the equation (10) numerically and have studied the effects of nonextensive electrons and positrons on the propagation of ion acoustic solitary waves. The wave phase velocity $\lambda$ given by equation (9) is found numerically using equation (8). In order to investigate the effect of nonextensivity on the wave phase velocity we have plotted $\lambda$ as a function of positron density (p) in Fig. 1(a) for three different values of nonextensive parameters q i.e. $q=1.0$, $\mathrm{q}=1.4$ and $\mathrm{q}=1.6$ with $\sigma=0.05$ and $u=0.1$. It is observed that phase velocity $\lambda$ decreases with positron density and nonextensivity for the range $q>1$. Similar behavior has been observed for other two ranges of nonextensivity i.e. $-1<q<0$ and $0<q<1$ (not shown). As $q=1$ corresponds to usual Maxwellian nature of the particles. So any deviation from Maxwellian behaviour leads to decrease in phase velocity of the soliton. This also becomes clear from the mathematical expression for phase velocity given by equation (9) where $\lambda$ is inversely related to $\mathrm{q}$.
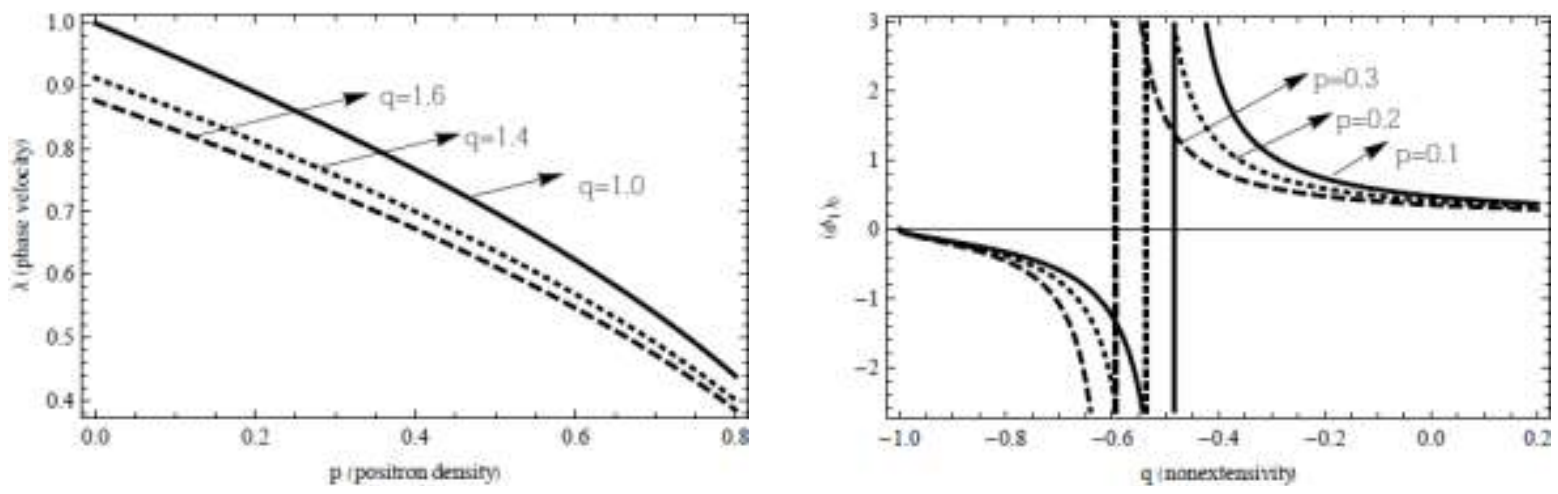

Fig. 1(a): Variation of wave phase velocity $\lambda$ with positron density $\mathrm{p}$ for $q=1.0$ (solid line), $q=1.4$ (dotted line) and $q=1.6$ (dashed line) with $\sigma=0.05$ and $u=0.1$.

Fig. 1(b): Variation of peak amplitude of planar solitary structures $\left(\phi_{1}\right)_{0}$ with nonextensive parameter q for $p=0.1$ (solid line), $p=0.2$ (dotted line) and $p=0.3$ (dashed line) with $\sigma=0.05$ and $\mathrm{u}=0.1$

For planar structures i.e. $\mathrm{m}=0$, Fig. 1(b) shows the variation of peak amplitude of solitary structures $\left(\phi_{1}\right)_{0}$ as a function of nonextensive parameter $\mathrm{q}$ for three different values of positron density $\mathrm{p}$. Here we have taken three values of positron density, $\mathrm{p}=0.1$ (solid line), $\mathrm{p}=0.2$ (dotted line) and $\mathrm{p}=0.3$ (dashed line) for the region $-1<q<0$. The other parameters are taken as $\sigma=0.05, u=0.1$ etc. It is observed that nonextensivity leads to transition from negative to positive potential solitary structures. It is further mentioned that this transition occurs earlier for larger values of positron density. Similar transition behaviour is also clear from the plot of soliton solution of planar solitary structures as a function of variable $\chi$ for the range $-1<q<0$ as is given in Fig. 2(a) for three different values of q. While only positive potential structures are obtained for the regions $0<q<1$ and $q>1$. It is mentioned that for all the ranges of q, the peak amplitude decreases with increase in q. This behaviour is depicted in Fig. 2(b), where a plot of planar soliton potential $\phi_{1}$ as a function of variable $\chi$ is given for the range $0<q<1$ for three different values of nonextensive parameters. 

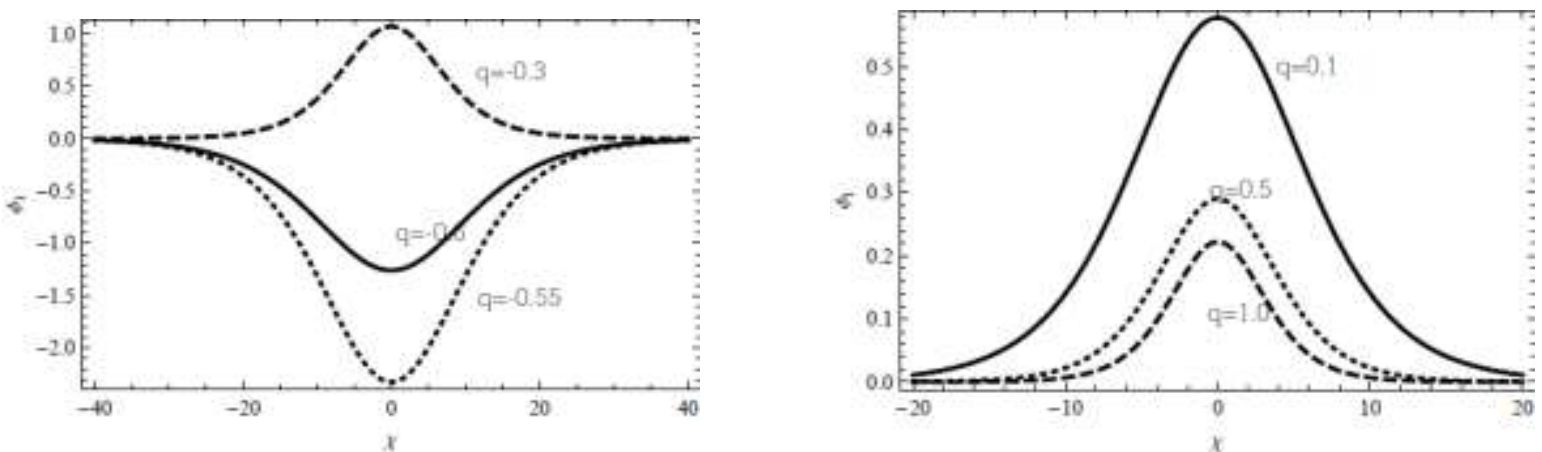

Fig. 2(a): For the range $-1<q<0$, variation of soliton solution $\phi_{1}$ as a function of variable $\chi$ with $\sigma=0.05$ and $\mathrm{u}=0.1$.

Fig. 2(b): Variation of soliton solution $\phi_{1}$ as a function of variable $\chi$ for the range $q>0$ with $\sigma=0.05$ and $\mathrm{u}=$ 0.1 .

In equation (10), $\frac{m}{2 \tau} \phi$ is a geometrical term and singular at $\tau \rightarrow 0$. For sufficiently large values of $|\tau|$, the spherical and cylindrical solitary wave reduces to planar waves. This is due to the fact that large $|\tau|$ makes the term $\frac{m}{2 \tau} \phi$ non-dominent. However for lower value of $\tau$, the geometrical term will be dominant and both spherical and cylindrical waves and will be different from the one dimensional solitary waves. In Fig. 3(a), the variation of the soliton solution of cylindrical ion acoustic solitary waves $\phi_{2}$ with respect to space coordinate $\xi$ for three different values of nonextensive parameter $\mathrm{q}$ has been given for the region $0<q<1$. Here solid line is for $\mathrm{q}=0.1$, dotted line id for $\mathrm{q}=0.2$ and dashed line is for $\mathrm{q}=0.3$. We observe that the cylindrical soliton amplitude goes on decreasing with increase in $\mathrm{q}$. This variation remains similar for the region $q>1$ (not shown here)
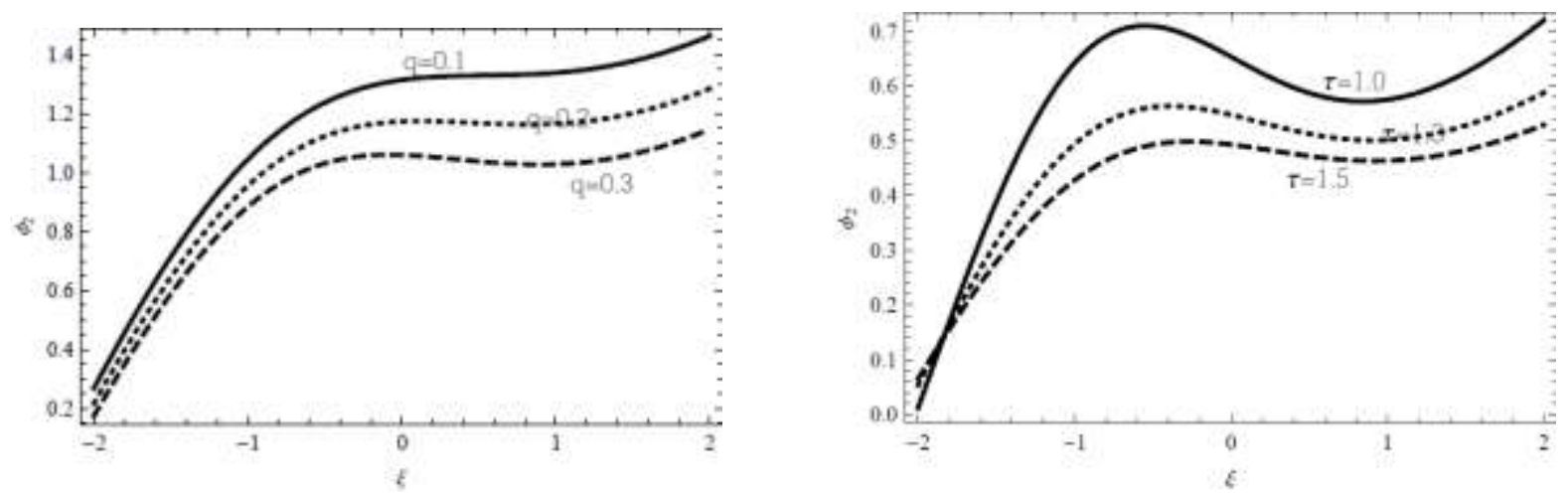

Fig. 3(a): Variation of cylindrical soliton solution $\phi_{2}$ as a function of space coordinate $\xi$ for three different values of $\mathrm{q}$ with $\sigma=0.1, \mathrm{u}=0.5, p=0.3$.

Figure 3(b): Variation of cylindrical soliton solution $\phi_{2}$ as a function of space coordinate $\xi$ for three different values of $\tau$ with $\sigma=0.1, \mathrm{u}=0.5, p=0.3$ and $\mathrm{q}=0.9$.

The solution $\phi_{2}$ for cylindrical geometry is given by equation (18) is valid for $\tau>0$. In Fig. 3(b), we take the variation of the soliton solution $\phi_{2}$ with respect to space coordinate $\xi$ for different values of time coordinate $\tau$ i.e. $\tau=1.0, \tau=1.3$ and $\tau=1.5$ for the region $0<q<1$. The other parameters are with $\sigma=$ $0.1, p=0.3, m=1, q=0.9$ and $u=0.5$. It is mentioned that their behaviour is similar to the one obtained by Sahu and Roychoudhury [42]. We also observed that the soliton amplitude goes on decreasing with increase in $\tau$. The observed solitary structures are of compressive nature or they are positive potential structures. It may be noted that this variation remains same for the region $q>1$.

The 3D profile for the variation of soliton solution of cylindrical geometry with as a function of $\xi$ and $q$ is given in Fig. 4(a). The other parameters are taken as $\sigma=0.1, p=0.3, u=0.5$ and $\chi=2$ for $q>1$. Here we observed the compressive solitons for the nonplanar geometry in case of $m=1$. If we compare these structures with the 3D profiles of planar structures, we have seen that the peaks of the nonplanar solitons are slightly moved away from the origin. While in planar cases the peaks are formed at the origin. For the range $q<0$, the three dimensional view of the soliton profile is given in Fig. 4(b). 

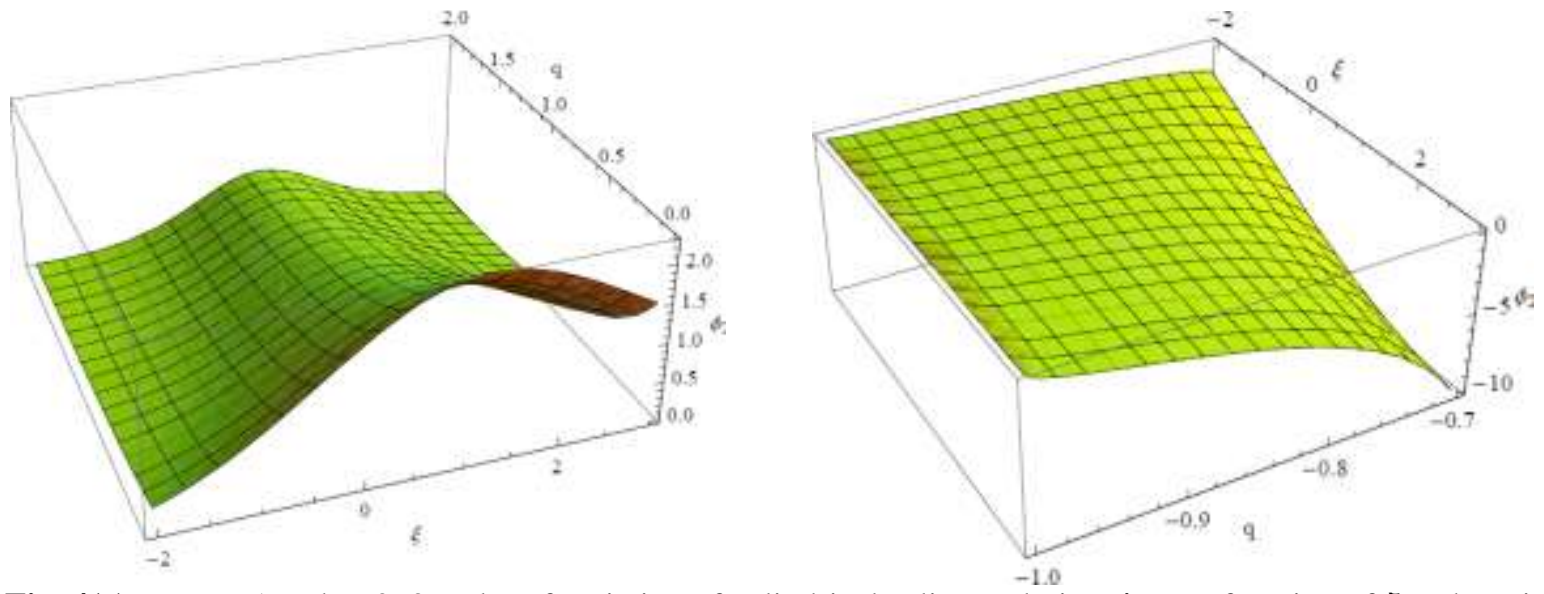

Fig. 4(a): For $m=1$ and $q>0,3 D$ plot of variation of cylindrical soliton solution $\phi_{2}$ as a function of $\xi$ and $q$ with $\sigma=0.1, p=0.3, \tau=1$ and $u=0.5$.

Fig. 4(b): For $m=1$ and lower values of $q, 3 D$ plot of variation of soliton solution $\phi_{2}$ as a function of $\xi$ and $q$ with $\sigma=0.1, p=0.3, m=1$ and $u=0.5$.

In last we give the comparison between the planar and nonplanar geometries. This comparison consists of the variation of the peak amplitudes of the planar as well as nonplanar geometries with respect to the nonextensive parameter $q$ for the ranges $-1<q<0$ and $q>1$ and is shown in figures 5(a) and 5(b) respectively.
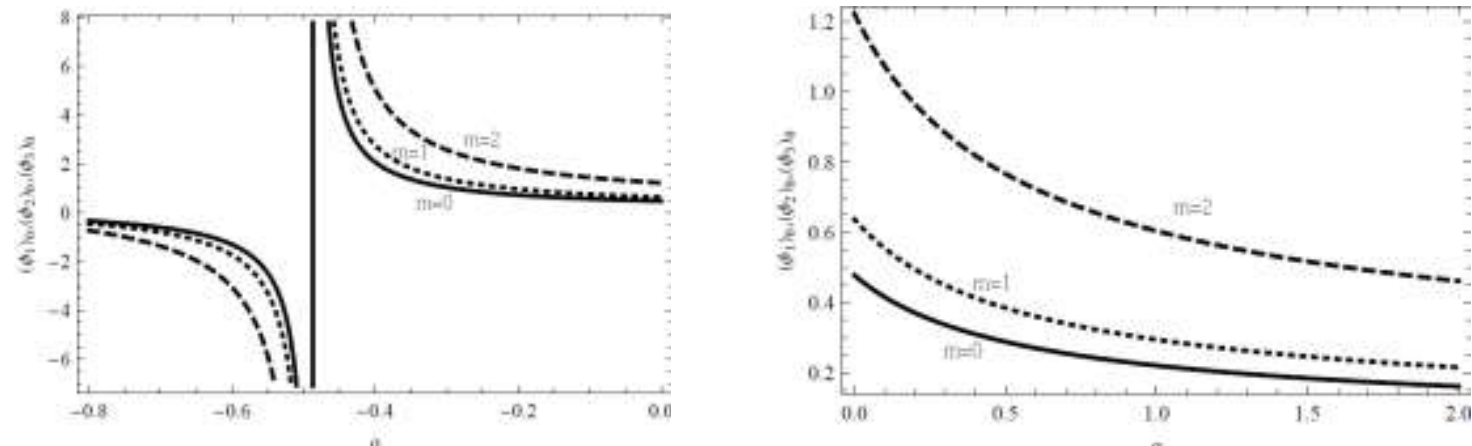

Fig. 5(a): Variation of peak amplitudes of flat geometry $\left(\phi_{1}\right)_{0}(\mathrm{~m}=0)$, cylindrical geometry $\left(\phi_{2}\right)_{0}(\mathrm{~m}=1)$ and spherical geometry $\left(\phi_{3}\right)_{0}(\mathrm{~m}=2)$ structures with $\mathrm{q}$ for $-1<q<0$ with $\sigma=0.1, p=0.1, \tau=2$ and $u=0.1$.

Fig. 5(b): Variation of peak amplitudes of flat geometry $\left(\phi_{1}\right)_{0}$, cylindrical geometry $\left(\phi_{2}\right)_{0}$ and spherical geometry $\left(\phi_{3}\right)_{0}$ structures with $\mathrm{q}$ (nonextensive parameter) for $q>1$ having $\sigma=0.1, p=0.1, \tau=2$ and $u=0.1$.

The set of parameters taken are mentioned in respective captions. For both the ranges of q, spherical solitary waves have maximum amplitude and planar waves has minimum. While the amplitude for cylindrical solitary waves lies between these two. This behaviour is similar to the one observed by Javidan [39] and Sahu and Roychoudhury [42]. However, for large values of $\tau$ the amplitude of cylindrical and spherical solitary waves become similar to the planar case. As mentioned earlier, this is because the nonplanar geometrical effects are no longer dominant for larger values of $\tau$. The presented investigation may helpful in better understanding of study of cylindrical and spherical solitary waves in astrophysical plasmas. The comparison of the obtained results with other particle distributions in spherical and cylindrical geometries can help is to find better knowledge in plasma physics.

\section{Conclusion}

The properties of spherical and cylindrical ion acoustic solitary waves (IASWs) have been studied in ep-i plasma consisting of ions, nonextensive electrons and positrons by considering Tsallis distributed electrons and positrons. Here we have observed that wave phase velocity $(\lambda)$, decreases with both positron density (p) and nonextensivity $(\mathrm{q})$ of particles. We found that a transition from negative (rarefactive) to positive (compressive) potential structures have been observed for all the three geometries for which peak amplitudes decreases with $\mathrm{q}$ 
for range $-1<q<0$, while only positive potential structures are observed for the ranges $0<q<1$ and $q>$ 1. For the same set of parameters, peak amplitude of spherical waves is maximum and planer waves are minimum while the peak amplitude of cylindrical waves lies between the two. It may be mentioned that the obtained results are similar to the one obtained by Javidan [39] and Sahu and Roychoudhury [42]. The presented investigation may helpful in better understanding of study of cylindrical and spherical solitary waves in astrophysical plasmas. The comparison of the obtained results with other particle distributions in spherical and cylindrical geometries can help is to find better knowledge in plasma physics.

\section{References}

[1] H. Ikezi, R. Taylor and D. Baker, Formation and interaction of ion-acoustic solitons, Physics Review Letters 25(1), $1970,11-14$.

[2] S. Bahamida, K. Annou and R. Annou, Ion-acoustic solitons in electron positron nonthermal plasma, $34^{\text {th }}$ EPS Conference on Plasma Physics, Warsaw, 31F, 2007, P-4.139.

[3] T. K. Baluku, Ion acoustic solitary waves in an electron positron plasma with nonthermal electrons, Plasma Physics Control Fusion 53(9), 2011, 095007.

[4] T. K. Baluku and M. A. Hellberg, Ion acoustic solitons in a plasma with two temperature kappa distributed electrons, Physics of Plasmas 19(1), 2012, 012106.

[5] N. Boubakour, M. Tribeche and K. Aoutou, Ion acoustic solitary waves in a plasma with superthermal electrons and positrons, Physica Scripta 79(6), 2009, 065503.

[6] R. A. Cairns, A. A. Mamun, R. Bingham and P. K. Shukla, Ion-acoustic solitons in a magnetized plasma with nonthermal electrons, Physica Scripta 1996(T63), 1996, 80-86.

[7] G. C. Das and S. N. Paul, Ion- acoustic solitary waves in relativistic plasma, Physics of Fluids 28(3), 1985, 823-825.

[8] G. C. Das and S. G. Tagare, Propagation of ion-acosutic waves in a multicomponent plasma, Plasma Physics 17(12), 1975, 10251032 .

[9] S. I. Popel, S. V. Vladimirov and P. K. Shukla, Ion-acoustic solitons in electron-positron-ion plasmas, Physics of Plasmas 2(3), 1995, 716-719.

[10] Q. Haque, Vortex structures in dense electron-positron-ion plasmas, Physica Scripta 80(5), $2009,055501$.

[11] S. Mahmood, A. Mushtaq and H. Saleem, Ion acoustic solitary wave in homogeneous magnetized electron positron ion plasmas, New Journal of Physics 5, 2003, 28.

[12] P. K. Shukla, J. T. Mendonca and R. Bingham, Low-frequency electromagnetic waves in a magnetized electron-positron-ion plasma, Physica Scripta T113, 2004, 133.

[13] A. Rahman, S. Ali, A. M. Mirza and A. Qamar, Planar and nonplanar ion acoustic shock waves in relativistic degenerate astrophysical electron-positron-ion plasmas, Physics of Plasmas 20(4), 2013, 042305.

[14] S. A. Shan, A. Mushtaq and N. Akhtar, Ion acoustic double layers in the presence of positrons beam and q-nonextensive velocity distributed electrons, Astrophysics and Space Science 348(2), 2013, 501-510.

[15] A. Shah and R. Saeed, Ion acoustic shock waves in a relativistic electron-positron-ion plasmas, Physics Letters A 373(45), 2009, 4164-4168.

[16] H. R. Pakzad, Ion acoustic shock waves in dissipative plasma with superthermal electrons and positrons, Astrophysics and Space Sci 331(1), 2011, 169-174.

[17] S. Hussain, H. Ur-Rehman and S. Mahmood, Two dimensional ion acoustic shocks in electron-positron-ion plasmas with warm ions, and q-nonextensive distributed electrons and positrons, Astrophysics and Space Sci 351(2), 2014, 573-580.

[18] S. Beskin and E. E. Nokhrina, The effective acceleration of plasma outflow in the paraboloidal magnetic field, Monthly Notices of the Royal Astronomical Society 367(1), 2006, 375-86.

[19] W. H. Lee, E. Ramirez-Ruiz and D. Page, Opaque or transparent? a link between neutrino optical depths and the characteristic duration of short gamma-ray bursts, The Astrophysical Journal Letters 608(1), 2004, L5-L8.

[20] M. Lipunov, The ecology of rotators, Astrophysics and space science 132(1), 1987, 1-51.

[21] F. C. Michel, Theory of pulsar magnetospheres, Reviews of Modern Physics 54(1), 1982, 1.

[22] M. C. Begelman, R. D. Blandford and M. J. Rees, Theory of extragalactic radio sources, Reviews of Modern Physics 56(2), 1984, 255 .

[23] B. Kozlovsky, R. J. Murphy and G. H. Share, Positron-emitter production in solar flares from 3he reactions, The Astrophysical Journal 604(2), 2004, 892-99.

[24] E. V. Derishev, F. A. Aharonian and V. V. Kocharovsky, Off-Axis Emission from Relativistic Plasma Flows, The Astrophysical Journal 655(2), 2007, 980-88.

[25] V. V. Zheleznyakov and S. A. Koryagin, Polarization spectra of synchrotron radiation and the plasma composition of relativistic jets, Astronomy letters 28(11), 2002, 727-44.

[26] V. V. Zheleznyakov and S. A. Koryagin, On the content of cold electrons in blazar and microquasar jets, Astronomy letters 31(11), 2005, 713-28.

[27] Ya. B. Zeldovich and I. D. Novikov, Relativistic Astrophysics 2: The structure and evolution of the universe (Chicago, University of Chicago Press, 1983).

[28] V. Berezhiani, D. D. Tskhakaya and P. K. Shukla, Pair production in a strong wake field driven by an intense short laser pulse, Physics Review A 46(10), 1992, 6608.

[29] E. P. Liang, C. W. Scott and M. Tabak, Pair Production by Ultraintense Lasers, Physics Review Letters 81(22), $1998,4887$.

[30] Y. I. Salamin, S. X. Hu, K. Z. Hatsagortsyan and C. H. Keitel, Relativistic high-power laser-matter interactions, Physics Reports 427(2), 2006, 41-155.

[31] C. M. Surko and T. J. Murphy, Use of the positron as a plasma particle, Physics of Fluids B 2(), $1990,1372$.

[32] C. M. Surko, M. Leventhal, W. S. Crane, A. Passner, F. Wysocki, T. J. Murphy, J. Strachan and W. L. Rowan, Use of positrons to study transport in tokamak plasmas, Review of Scientific Instruments 57(8), 1986, 1862.

[33] C. Tsallis, Possible generalization of Boltzmann-Gibbs Statistics, Journal of Statistical Physics 52(1), $1988,479-487$.

[34] A. Renyi, On a new axiomatic theory of probability, Acta Mathematica Hungarica 6(3-4), 1955, $285-335$.

[35] P. Eslami, M. Mottaghizadeh and H. R. Pakzad, Nonplanar ion-acoustic solitary waves in electron-positron-ion plasmas with electrons following a $q$-nonextensive distribution, Physica Scripta 83(6), 2011, 065502.

[36] M. Ferdousi, S. Yasmin, S. Ashraf and A. A. Mamun, Nonlinear propagation of ion-acoustic waves in an electron-positron-ion plasma, Astrophysics and Space Science 352(2), 2014, 579-584. 
[37] M. G. Hafez, M. R. Talukder and R. Sakthivel, Ion acoustic solitary waves in plasmas with nonextensive distributed electrons, positrons and relativistic thermal ions, Indian Journal of Physics 90(5), 2016, 603-611.

[38] S. Maxon, Cylindrical and spherical solitons, Journal of Mathematics 8(1-2), 1978, 269-282.

[39] K. Javidan, Cylindrical and spherical ion acoustic solitary waves in electron-positron-ion plasmas with superthermal electrons, Astrophysics and Space Science 343(2), 2013, 667-673.

[40] N. Jehan, S. Mahmood and A. M. Mirza, Cylindrical and spherical ion-acoustic solitons in adiabatically hot electron-positron-ion plasmas, Physica Scripta 76(6), 2007, 661.

[41] R. Sabry, W. M. Moslem and P. K. Shukla, Planar and nonplanar ion-acoustic envelope solitary waves in a very dense electronpositron-ion plasma, European Physical Journal D 51(2), 2009, 233-240.

[42] B. Sahu and R. Roychoudhury, Cylindrical and spherical ion acoustic waves in a plasma with nonthermal electrons and warm ions, Physics of Plasmas 12(5), 2005, 052106.

[43] P. Eslami and M. Mottaghizadeh, Cylindrical ion-acoustic solitary waves in electronegative plasmas with superthermal electrons, Physics of Plasmas 19(6), 2012, 062110.

[44] P. Goldreich and W. H. Julian, Pulsar electrodynamics, Astrophysical Journal 157(2), 1969, 869-80.

[45] H. R. Miller and P. J. Witta, Active Galactic Nuclei (Springer, Berlin, 1987).

[46] R. Schlickeiser and P. K. Shukla, Cosmological magnetic field generation by the Weibel instability, Astrophysical Journal Letters 599(2), 2003, L57.

[47] P. Lightman, Relativistic thermal plasmas - pair processes and equilibria, Astrophysical Journal 253(1), 1982, 842-58.

[48] M. L. Burns and R. V. E. Lovelace, Theory of electron-positron showers in double radio sources, Astrophysical Journal 262(1), 1982, 87-99.

[49] A. P. Lightman and A. A. Zdziarski, Pair production and Compton scattering in compact sources and comparison to observations of active galactic nuclei, Astrophysical Journal 319(1), 1987, 643-61.

[50] A. A. Zdziarski, Saturated pair-photon cascades on isotropic background photons, Astrophysical Journal 335(1), 1988, 786-802.

[51] P. K. Shukla, N. N. Rao, M. Y. Yu and N. L. Tsintsadze, Relativistic nonlinear effects in plasmas, Physics Reports 138(1-2), 1986, $1-149$.

[52] P. Helander and D. J. Ward, Positron Creation and Annihilation in Tokamak Plasmas with Runaway Electrons, Physics Review Letters 90(13), 2003, 135004.

[53] Tandberg, E. Hansen and A. G. Emslie, The Physics of Solar Flares (Cambridge, University Press, 1988).

[54] F. B. Rizzato, Weak nonlinear electromagnetic waves and low-frequency magnetic-field generation in electron-positron-ion plasmas, Plasma Physics 40(2), 1988, 289-98.

[55] M. Y. Yu, P. K. Shukla and L. Stenflo (1986) Alfven vortices in a strongly magnetized electron-positron plasma, Astrophysical Journal 309(2), 1986, L63-L66.

[56] S. Jammalamadaka, P. K. Shukla and L. Stenflo, Vortices in strongly magnetized nonuniform electron-positron-ion plasmas, Astrophysics and Space Science 240(1), 1996, 39-43.

[57] P. K. Shukla, R. Fedele and L. Stenflo, Nonlinear effects caused by intense electromagnetic waves in an electron-positron-ion plasma, Physics of Plasmas 10(1), 2003a, 310-13.

[58] P. K. Shukla, A. A. Mamun and L. Stenflo, Vortices in a Strongly Magnetized Electron-Positron-Ion Plasma, Physica Scripta 68(4), 2003b, 295.

[59] Kourakis, F. Verheest and N. Cramer, Nonlinear perpendicular propagation of ordinary mode electromagnetic wave packets in pair plasmas and electron-positron-ion plasmas, Physics of Plasmas 14(2), 2007, 022306.

[60] R. Sabry, W. M. Moslem and P. K. Shukla, Planar and nonplanar ion-acoustic envelope solitary waves in a very dense electronpositron-ion plasma, European Physical Journal D 5(2), 2009, 233-40.

[61] E. I. El-Awady, S. A. El-Tantawy, W. M. Moslem and P. K. Shukla, Electron-positron-ion plasma with kappa distribution: Ion acoustic soliton propagation, Physics Letters A 374(31-32), 2010, 3216-19.

[62] M. Akbari-Moghanjoughi, Effects of ion-temperature on propagation of the large-amplitude ion-acoustic solitons in degenerate electron-positron-ion plasmas, Physics of Plasmas 17(8), 2010, 082315.

[63] A. Shah, R. Saeed and M. Noaman-ul-Haq, Nonplanar converging and diverging shock waves in the presence of thermal ions in electron-positron plasma, Physics of Plasmas 17(7), 2010, 072307.

[64] S. Maxon and J. Viecelli, Spherical solitons, Physics Review Letters 32(1), 1974, 4-6.

[65] A. Sahu and R. Roychoudhury, Exact solutions of cylindrical and spherical dust ion acoustic waves, Physics of plasmas 10(10), $2003,4162$. 